\title{
Literaturbericht
}

\author{
Oliver Schwarz
}

\section{Same but different: Externe Europäisierung zwischen Beitritt, Nachbarschaft und Kooperation}

Neben „Integration“ zählt „Europäisierung“ heute zu den zentralen Kategorien der politikwissenschaftlichen Forschung zur Europäischen Union (EU). Diese konzentrierte sich lange Zeit vornehmlich auf die Beschreibung des europäischen Einigungsprozesses und die Erklärung des hieraus resultierenden Staatenverbundes. Mitte der 1990er Jahre hat sich die Forschung dann verstärkt mit der Rückwirkung der europäischen Integration auf die EU-Mitgliedsstaaten auseinandergesetzt. Hierbei stand die Frage im Vordergrund, unter welchen Bedingungen sich Veränderungen in den Inhalten, Strukturen und Prozessen nationaler Politik vollziehen und inwieweit diese auf den europäischen Integrationsprozess zurückzuführen sind. Wissenschaftler verschiedener Provenienz haben sich die Europäisierungsforschung als neues Betätigungsfeld erschlossen und eine kaum zu überblickende Anzahl von Publikationen zu den unterschiedlichsten Facetten dieses Themas veröffentlicht. Dies erschwert die Auswahl der zu behandelnden Beiträge für einen Literaturbericht. Der gesetzte Rahmen wird schnell gesprengt und ohne entsprechende Strukturierung und Eingrenzung droht die Darstellung skizzenhaft zu bleiben. Um die Fülle der vorhandenen Literatur einzugrenzen, blendet dieser Literaturbericht Arbeiten zur Europäisierung von EU-Mitgliedsstaaten explizit aus. Berücksichtigt werden lediglich Beiträge, die sich mit der Europäisierung von EU-Drittstaaten beschäftigen. Freilich sind die Übergänge zwischen interner und externer Europäisierung fließend und nicht immer klar voneinander zu unterscheiden.

Der Literaturbericht ist wie folgt gegliedert: In einem ersten Abschnitt wird anhand der EU-Osterweiterung aufgezeigt, wie sich das Konditionalitätstheorem zum dominierenden Erklärungsansatz der Europäisierungsforschung entwickelt und auch im Falle des Beitrittes von Bulgarien und Rumänien nicht an Plausibilität verloren hat. Hieran schließt sich die Frage an, inwieweit sich die Europäisierung der Staaten Südosteuropas von der bislang seitens der EU verfolgten Erweiterungspolitik unterscheidet. Im zweiten Abschnitt rücken Europäisierungsprozesse im Rah- 
men der Europäischen Nachbarschaftspolitik (ENP) in den Vordergrund. In einem dritten Abschnitt wird den Grenzen und Möglichkeiten der externen Europäisierung „beyond Europe“" nachgegangen und Anknüpfungspunkte an das Modell einer EU als „normative power Europe“ aufgezeigt. Der Literaturbericht schließt mit einem Ausblick auf zukünftige empirische Forschungen und theoretische Weiterentwicklungen der Europäisierung von EU-Drittstaaten.

\section{EU-Osterweiterung: Das Big-Bang-Theorem der Konditionalität}

In einem gemeinsamen Beitrag haben Frank Schimmelfennig und Ulrich Sedelmeier kritisch angemerkt, dass es sich bei der EU-Erweiterung aus integrationstheoretischer Perspektive um einen eher vernachlässigten Untersuchungsgegenstand handelt (2002: 501). Dies muss auf den ersten Blick irritieren, da seit dem Beginn der europäischen Entwicklungsgeschichte im Prinzip nur drei mehr oder weniger kurze Zeiträume existierten, in denen sich der Integrationsverbund nicht mit Beitrittsgesuchen von Drittstaaten auseinanderzusetzen hatte. Erweiterung gehört somit offensichtlich zu einem integralen Bestandteil des europäischen Einigungsprozesses. Dennoch blieb die Europaforschung eine systematische Aufarbeitung dieses Themenfeldes lange Zeit schuldig. Gerade die Rückwirkungen des Erweiterungsprozesses auf die der EU beitretenden Drittstaaten wurden weitgehend ausgeblendet.

Dankenswerterweise beließen es die Autoren nicht dabei, lediglich auf diese eklatante Forschungslücke hinzuweisen, sondern leisteten selbst einen kaum zu überschätzenden Beitrag zur Überwindung derselben. In ihrem viel zitierten Sammelband „The Europeanization of Central and Eastern Europe“ (Schimmelfennig/ Sedelmeier 2005 a) werden am Beispiel ausgewählter Staaten Mittel- und Osteuropas drei verschiedene Erklärungsmodelle empirisch getestet. Schimmelfennig und Sedelmeier kommen in einem zusammenfassenden Beitrag zu dem Schluss, dass das sogenannte „external incentives model“ am ehesten geeignet ist, die vielzähligen Prozesse der Normanpassung in den Kandidatenstaaten zu erklären. Die Grundannahme dieses Modells lautet mit den Worten der Autoren: „A government adopts EU rules if the benefits of EU rewards exceed the domestic adaption costs" (dies. 2005 b: 12). Als wesentliche Voraussetzungen werden dabei die Determiniertheit der Bedingungen, die Größe und Zeitnähe der Anreize, die Glaubwürdigkeit der Konditionalität und die Größe der Anpassungskosten genannt. Unter den genannten Faktoren nimmt aus Sicht der Autoren das Konzept der Konditionalität eine zentrale Erklärungsfunktion ein: ,[...] a key insight that we derive from the contributions is that the influence of the EU depends crucially on the context in which the EU uses 
its incentives. We thus suggest distinguishing between the context of democratic conditionality and the context of acquis conditionality" (ebd.: 28).

Mit ihren empirischen Ergebnissen zur Bedeutung der Konditonalität haben die beiden Autoren ein zentrales Theorem der Europäisierungsforschung entwickelt, das in der Literatur eine breite Rezeption gefunden hat. Eine treffende Definition der EU-Konditionalität findet sich in den Arbeiten von Bernard Steunenberg und Antoaneta Dimitrova: „We define EU enlargement conditionality as an exchange between the EU and a candidate country in which the EU offers the candidate a (realistic) prospect of EU membership, if the candidate implements a wide range of (EU driven) domestic reforms. The so called carrot and stick approach of conditionality involves the withdrawal of the benefits of accession and halting or slowing down the process, if candidate states' governments fail to progress with reforms“ (2007: 3). Zwar hat die Europäisierungsforschung eine Reihe weiterer Mechanismen identifiziert, über die die EU einen nationalen Wandel in Kandidatenstaaten zu initiieren vermag, keinem dieser Mechanismen wurde jedoch eine derartige Wirksamkeit attestiert wie dem Prinzip der Konditionalität. Heather Grabbe konstatiert diesbezüglich: „The most important mechanism is the EU's gate-keeping role in determining when each candidate can progress to the next stage towards accession" (2001: 1019 f.). Im Verlauf des Gate-keeping sind die Kandidatenstaaten nur bedingt in der Lage, eigene Forderungen in den Verhandlungsprozess einzubringen und durchzusetzen. Bedingung eines EU-Beitritts und damit antizipiertes Ergebnis des Europäisierungsprozesses ist die uneingeschränkte Übernahme des Acquis communautaire durch den neuen Mitgliedsstaat. Dies ist das wesentliche Charakteristikum, das die „Beitrittseuropäisierung“ von der „Mitgliedseuropäisierung" unterscheidet (Axt/Milošoski/Schwarz 2007: 142).

\section{Bulgarien und Rumänien: Die Erfindung der Post-Beitrittskonditionalität}

Trotz der asymmetrischen Struktur der Beitrittseuropäisierung existieren zur Übernahme des Acquis offensichtlich Verhandlungsspielräume. Dies belegen die rund 300 Übergangsregelungen, die den mittel- und osteuropäischen Staaten beim Vollzug ihres EU-Beitritts eingeräumt wurden (Avery 2004: 56; Lippert 2004: 32). Die meisten dieser Regelungen betrafen den Gemeinsamen Markt, die Eurozone und den Schengener Raum. Mit dem EU-Beitritt Bulgariens und Rumäniens 2007 erlebte das Instrument der Konditionalität jedoch eine grundlegende Veränderung. In einem gemeinsamen Beitrag zeichnen Gergana Noutcheva und Dimitar Bechev nach, dass die „Vorbeitrittskonditionalität“ (2008: 140) der EU im Falle Bulgariens und Rumäniens nicht in der Lage war, rechtzeitig zum Beitritt bestehende defizitäre 
Strukturen zu verändern und notwendige Veränderungen gegenüber innenpolitischen Vetoplayern zu initiieren. Anstatt diesen Europäisierungsrückstand mit einer Verschiebung des Beitrittstermins zu sanktionieren, wurde für Bulgarien und Rumänien ein nachträgliches Monitoringsystem geschaffen, um mögliche negative Rückkopplungen auf den europäischen Integrationsverbund zu verhindern und den grundsätzlichen Wirkmechanismus der Konditionalität auch über den Status der EU-Mitgliedschaft auszuweiten (Pridham 2007). Eli Gateva (2010) hat diesen Sachverhalt treffenderweise mit der Begrifflichkeit der „Post-Beitrittskonditionalität“ umrissen.

Ähnlich wie bei den Staaten Mittel- und Osteuropas sahen die mit Bulgarien und Rumänien vereinbarten Beitrittsverträge in politisch sensiblen Bereichen wie denen des Binnenmarkts und der Wirtschaft eine Reihe von Schutzklauseln vor. Handelte es sich hierbei noch um übliche handelspolitische Schutzmaßnahmen, wurde für den Bereich Justiz und Inneres ein gänzlich neuartiges Verfahren eingeführt: das sogenannte Kooperations- und Kontrollverfahren (Markov 2010). Im Rahmen dieses Verfahrens wurden für Rumänien vier und für Bulgarien insgesamt sechs Benchmarks definiert. Diese Benchmarks enthalten für beide Länder eine Reihe konkreter Vorgaben, die insbesondere die Verbesserung von Transparenz und Effizienz der Gerichtsverfahren, die Fortsetzung von Reformen im Justizwesen und Maßnahmen zur Prävention und Bekämpfung von Korruption betreffen. Im Falle Bulgariens wurden explizit auch Verfassungsänderungen angemahnt, um jegliche Zweifel an der Unabhängigkeit und Rechenschaftspflicht des Justizwesens auszuräumen und eine Strategie zur Bekämpfung des organisierten Verbrechens voranzutreiben. Mit Beginn ihrer EU-Mitgliedschaft mussten beide Länder jährlich Rechenschaft über die Erfüllung ihrer Benchmarks ablegen; die Fortschritte wurden von der Europäischen Kommission alle sechs Monate in Berichten zusammengefasst. Kam die Kommission hierbei zu dem Urteil, dass den gesetzten Vorgaben nicht angemessen nachgekommen wurde, so konnte sie die in den Beitrittsverträgen fixierten Schutzmaßnahmen anwenden. Die Kommission veröffentlichte ihre ersten beiden Fortschrittsberichte im Juni 2007. Trotz der nicht unbedingt befriedigenden Schlussfolgerungen wurden zunächst keine Sanktionen beschlossen. Dies änderte sich im Juli 2008. Während Rumänien lediglich mit deutlichen Worten ermahnt wurde, entzog die Kommission zwei bulgarischen Behörden die Zulassung und verwehrte Sofia den Zugriff auf europäische Finanzhilfen in dreistelliger Millionenhöhe.

Diese Entwicklungen veranlassten Florian Trauner zu der Überlegung, hinsichtlich der Implementation des Acquis könnten Bulgarien und Rumänien der sogenannten ,world of dead letters“ angehören (2009: 12). Der Terminus entstammt der 
Compliance-Forschung, die in ihrer europapolitischen Ausrichtung primär die rechtliche und administrative Umsetzung von Richtlinien und Verordnungen in verschiedenen Politikfeldern untersucht. Gerda Falkner u. a. (2005) haben diesbezüglich die drei „worlds of compliance“ definiert, die sich aus der ,world of law observance“, der ,world of domestic politics” und der ,world of neglect“ zusammensetzen. In einem späteren Beitrag hat die Autorin zusammen mit Oliver Treib noch eine zusätzliche Kategorie geschaffen: die sogenannte „world of dead letters“ (Falkner/Treib 2007). Diese vierte Welt zeichnet sich durch politisierte Umsetzungsprozesse und systematische Probleme bei der praktischen Anwendung und beim Vollzug europäischen Rechts aus.

Einen gravierenden Rückfall bei der demokratischen Konsolidierung Bulgariens und Rumäniens konnten nachfolgende Studien jedoch nicht ausmachen (Levitz/ Pop-Eleches 2009, 2010). Aneta Spendzharova und Milada Vachudova zeichnen stattdessen nach, dass beide Regierungen den spezifischen Anforderungen der Fortschrittsberichte der Kommission zwar unterschiedlich nachgekommen sind, die jeweiligen praktischen Reformanstrengungen jedoch ohne das Kooperations- und Kontrollverfahren deutlich weniger stark ausgefallen wären (2012: 55). Gerade im Falle Bulgariens habe die EU durch das Verhängen von Sanktionen dazu beigetragen, auch außerhalb der Regierung reformwillige Kräfte zu unterstützen und als Opposition zu stärken. Bei der Parlamentswahl im Juli 2009 zählte die Korruptionsbekämpfung zu einem der Hauptthemen des bulgarischen Wahlkampfes und war maßgeblich für die Niederlage der amtierenden Regierung verantwortlich. Eine derart wirksame Verknüpfung zwischen externen Einflüssen und nationalen Entwicklungen fand in Rumänien hingegen zunächst nicht statt. Erst mit der Erhöhung des Drucks durch die Kommission auf Bukarest im Verlauf des Jahres 2010 und die zunehmende Koppelung von Fortschritten im Bereich der Korruptionsbekämpfung an eine Teilnahme im Schengener Raum seien die rumänischen Reformbemühungen wieder intensiviert worden. Die Autorinnen kommen daher zu dem Schluss: "We hypothesise that political leaders and parties will only continue and deepen reforms in response to the twin forces of EU and domestic influence" (ebd.: 41).

Bei entsprechenden nationalen Akteurskonstellationen zeigt das Konditionalitätsmodell somit auch nach dem Vollzug des EU-Beitritts beachtliche Reformergebnisse. Vor diesem Hintergrund muss es ein wenig verwundern, dass die Kommission bereits angekündigt hat, das Modell des Kooperations- und Kontrollverfahrens nicht auf andere Beitrittskandidaten zu übertragen. Im Falle Kroatiens hat die EU beispielsweise explizit auf ein derartiges Prozedere verzichtet. Unabhängig davon wird die Kommission jedoch noch im Verlauf des Jahres 2012 eine Gesamt- 
bewertung der mit diesem Instrument gesammelten Erfahrungen vornehmen und entsprechende Empfehlungen für ein weiteres Vorgehen formulieren.

\section{Südosteuropa: Das doppelte Dilemma mangelnder Glaubwürdigkeit und begrenzter Staatlichkeit}

Mit der Aufnahme Bulgariens und Rumäniens hat die EU ihre Erweiterungspolitik jedoch keinesfalls eingestellt: Kroatien steht unmittelbar davor, dem Integrationsverbund als neues Mitgliedsland beizutreten. Bereits seit Oktober 2005 verhandelt die EU mit der Türkei über einen Beitritt. Gleichzeitig wurde auch Mazedonien der offizielle Status eines Beitrittskandidaten verliehen; Montenegro folgte im Dezember 2011. Zuletzt wurde Serbien im März 2012 der Kandidatenstatus verliehen. Während Albanien bereits seit April 2009 auf diesen Schritt wartet, mehren sich die Anzeichen, dass auch Bosnien-Herzegowina und Kosovo sich nicht weiterhin damit begnügen wollen, lediglich ,potenzielle“ Kandidaten für einen EU-Beitritt zu sein. Mit der Ausnahme Islands entstammen somit alle derzeitigen Beitrittskandidaten und Staaten mit einer Beitrittsperspektive dem geografischen Raum Südosteuropas (Noutcheva/Aydin-Düzgit 2012).

Während die Türkei ihrerseits schon im Juli 1959 ein Beitrittsgesuch stellte, auf das die damalige Gemeinschaft der Sechs im September 1963 mit einem Assoziierungsabkommen reagierte, bettete die EU den westlichen Balkan erst im Februar 1996 in ihren sogenannten Regionalansatz ein. Mit diesem Ansatz wollte die EU zur Umsetzung des 1995 geschlossenen Daytoner Abkommens beitragen und die regionale Zusammenarbeit zwischen den Westbalkanstaaten fördern. Hierzu sah der Regionalansatz den Abschluss vertraglicher Abkommen zwischen der EU und den betreffenden Staaten sowie Programme zur finanziellen Zusammenarbeit vor. Im April 1997 wurde vom Rat der EU eine Reihe von Bedingungen definiert, die als Voraussetzung für eine Zusammenarbeit mit der EU galten. Das Prinzip der Konditionalität zog somit in die Balkanpolitik der EU ein (Altmann 1998). Doch erst nach schmerzlichen Lernprozessen im Zuge des Kosovokrieges hat die EU im Juli 1999 mit der Initiierung des Stabilitätspakts für Südosteuropa einen Paradigmenwechsel von der bloßen Stabilisierung der Region hin zur Integration derselben vollzogen (Biermann 2002). Die erstmals über den Stabilitätspakt offerierte Perspektive einer EU-Mitgliedschaft stellt bis heute den zentralen Motivationsimpuls für die Länder des westlichen Balkans dar, ihre internen Reformanstrengungen zu intensivieren und eine regionale Kooperation voranzutreiben. Im Juni 2000 betonte der Europäische Rat in Feira dann erstmals, dass die Länder des westlichen Balkans „potenzielle Kandidaten“ für eine EU-Mitgliedschaft seien; eine Formulierung, die 
drei Jahre später auf dem Europäischen Rat von Thessaloniki und in später nachfolgenden Gipfelerklärungen ausdrücklich bestätigt wurde. Doch bislang haben sich die damit verbundenen Hoffnungen nur teilweise erfüllt, ja die Wirksamkeit der Erweiterung muss in der Region des westlichen Balkans sogar in Frage gestellt werden.

Doch worin unterscheidet sich der Europäisierungsprozess der Westbalkanstaaten von der bislang seitens der EU verfolgten Erweiterungspolitik? Tanja Börzel führt die Ursache der sich verhältnismäßig schleppend vollziehenden Europäisierung primär auf die begrenzte Staatlichkeit der Westbalkanstaaten zurück. Sie schlussfolgert: „Limited statehood seems to be the main cause of ineffective implementation of EU-induced reforms and the decoupling between formal institutional changes and rule-inconsistent behavior. It does not only affect the capacity to comply with EU expectations for domestic change but has also implications for the willingness of incumbent elites to adopt and implement reforms in the first place" (Börzel 2011:9). Unter den Staaten des westlichen Balkans trifft diese Einschätzung insbesondere auf Bosnien-Herzegowina und das Kosovo zu. Letzteres erklärte zwar im Februar 2008 seine Unabhängigkeit von Serbien, der völkerrechtliche Status des Landes ist seither jedoch umstritten. Auch Bosnien-Herzegowina muss mehr als 16 Jahre nach Kriegsende unverändert als internationales Protektorat bezeichnet werden. Von funktionierender Staatlichkeit kann in beiden Fällen somit nicht die Rede sein. Hierdurch erhält jedoch der Beitrittsprozess der Westbalkanstaaten bis hin zur Mitgliedschaft einen ungewissen Zeithorizont. Die Vagheit dieses Prozesses stellt die Glaubwürdigkeit der von der EU offerierten Beitrittsperspektive in Frage und konterkariert damit wiederum den Europäisierungsprozess der Beitrittsaspiranten. Die Literatur hat diesbezüglich auf die Notwendigkeit zusätzlicher politischer Angebote seitens der EU verwiesen, um kurzfristigen Ermüdungserscheinungen des Erweiterungsprozesses entgegenzuwirken (Steunenberg/Dimitrova 2007: 10; Lippert 2011). Im Falle der westlichen Balkanstaaten würde die EU diesen Ländern also zusätzliche politische Angebote unterbreiten, um die Glaubwürdigkeit der europäischen Beitrittsperspektive weiterhin aufrecht zu erhalten und um die Konsolidierung der dortigen Transformationsprozesse nicht zu gefährden. Ein praktischer Beleg für diese Politik der „flankierenden Überbrückung“ (Schwarz 2010) ist die von der EU in den letzten Jahren forcierte Liberalisierung ihres Visa-Regimes.

Die Glaubwürdigkeit der EU-Beitrittsperspektive wird jedoch nicht nur durch die vergleichsweise ungünstigen internen Strukturbedingungen der westlichen Balkanstaaten negativ beeinträchtigt, sondern auch durch die zunehmend innerhalb der EU zu konstatierende Erweiterungsmüdigkeit (Altmann 2005). So weicht der bislang innerhalb der EU vorherrschende permissive Konsens zur Fortführung der Erwei- 
terung einer sukzessiven Politisierung. Nichts belegt dies so deutlich wie die Diskussion um einen möglichen EU-Beitritt der Türkei (Börzel/Soyaltin 2012). Christophe Hillion (2010) spricht sogar von einer ,schleichenden Nationalisierung“ des Erweiterungsprozesses der EU. Der sukzessiv zunehmende Einfluss nationaler Partikularinteressen der Mitgliedsstaaten habe der Glaubwürdigkeit der EU schweren Schaden zugefügt. Hiermit sei nicht nur die Legitimität der EU-Konditionalität, sondern die Effektivität der europäischen Transformationsagenda als solche in Frage gestellt. In der Tat zeigt Dominik Tolksdorf (2011) am Beispiel der zähen Verhandlungen über eine Polizeireform in Bosnien-Herzegowina deutlich auf, dass die EU nicht immer kohärent gegenüber den Staaten des westlichen Balkans agiert. Zum Einfluss der Europäisierung hält der Autor zusammenfassend fest: „Durch die Bemühungen der EU, in Bosnien mit Hilfe von unklar definierten EU-Standards die ,Europäisierung ' des Landes voranzubringen, hat die EU-Konditionalität an Glaubwürdigkeit verloren“ (446). Wie die politisch verantwortlichen Akteure innerhalb der EU auf dieses Dilemma reagieren, bleibt ein ungebrochen spannendes Forschungsfeld. Nicht umsonst hält Tanja Börzel daher wie folgt fest: „The rather unfavorable domestic scope conditions render the Western Balkans a formidable test case for Europeanization approaches with their emphasis on membership conditionality shared by policy-makers in the EU“ (Börzel 2011: 6).

\section{Nachbarschaft: Formale Europäisierung durch differenzierte Konditiona- lität}

Bereits parallel zur Osterweiterung hat die EU mit der Europäischen Nachbarschaftspolitik (ENP) einen Politikansatz entwickelt, der die Beziehungen zu einer Reihe von Drittstaaten systematisch vertiefen sollte (Böttger 2010). Während sich ursprüngliche Initiativen noch vorwiegend auf Moldau, die Ukraine und Weißrussland konzentrierten, schloss die ENP schnell auch die Staaten des südlichen Mittelmeerraums sowie den sogenannten Barcelona-Prozess mit ein. Mit Armenien, Aserbaidschan und Georgien kamen schließlich auch die drei Länder des Südkaukasus hinzu. In ihrer heutigen Ausgestaltung umfasst die ENP derzeit insgesamt 17 Partnerstaaten. Die Beziehungen der EU zu Russland firmieren explizit nicht unter dem Dach der ENP. Stattdessen haben beide Seiten eine sogenannte Strategische Partnerschaft geschlossen, die auf der Realisierung von ,vier gemeinsamen Räumen" basiert.

In ihrer ursprünglichen Ausrichtung versteht sich die ENP explizit als Alternativangebot zur Erweiterung, das heißt die Übertragung europäischer Normen wird nicht durch die Gewährung einer Perspektive auf EU-Mitgliedschaft forciert (La- 
venex 2004). Gerade in ihrer politischen Finalität und praktischen Ausgestaltung ist die ENP unter den Akteuren der EU jedoch höchst umstritten (Lippert 2007, 2008). Auf den Wegfall der klassischen Beitrittskonditionalität hat die wissenschaftliche Literatur primär mit einer Hinwendung zu Ansätzen aus der Governance-Forschung reagiert (Lavenex 2008; Lavenex/Schimmelfennig 2009). Andrea Gawrich, Inna Melnykovska und Rainer Schweickert $(2009,2010)$ prägten den Begriff der „Nachbarschaftseuropäisierung“. Aufgrund des fehlenden Mechanismus der Konditionalität wurde die grundsätzliche Effektivität der Nachbarschaftseuropäisierung jedoch vielfach in Frage gestellt. Andrea Franke u. a. (2010) haben dazu beigetragen, dieses Urteil ein wenig zu differenzieren, indem sie zwischen verschiedenen Vorgehensweisen der EU im Rahmen der ENP differenzierten. So setzte die EU in einer frühen Phase der ENP allein auf eine bilaterale Strategie. Den Kern dieser Vorgehensweise bildeten hierbei die mit den Nachbarstaaten verhandelten Aktionspläne. In diesen Aktionsplänen wurden die jeweils länderspezifischen zentralen Prioritäten vertraglich festgelegt, die innerhalb eines Zeitraums von mehreren Jahren im Mittelpunkt der gegenseitigen Beziehungen stehen sollen. Mittlerweile sind für beinahe alle Staaten der ENP bilaterale Aktionspläne erstellt worden und in Kraft getreten.

In Analogie zum westlichen Balkan ging die EU in den vergangenen Jahren verstärkt zu einem regionalen Ansatz im Rahmen der ENP über. Die Regionalisierung begann zunächst mit der im Mai 2007 von der Kommission initiierten „Schwarzmeersynergie“, die die unmittelbare Nachbarschaft im Gebiet des Schwarzen Meeres umfasst und explizit auch Russland und die Türkei mit einschließt. Im Mai 2008 wurde auf polnische Initiative hin die „Östliche Partnerschaft“ ins Leben gerufen, die sich an insgesamt sechs östliche Nachbarstaaten der EU richtet. Zuletzt wurde im Juli 2008 unter der französischen Präsidentschaft die „Union für das Mittelmeer“ aus der Taufe gehoben, der die Mittelmeer-Anrainerstaaten sowie die an diese angrenzenden Staaten Jordanien und Mauretanien angehören.

Das ursprünglich diffuse Angebot der EU - ,everything but institutions“ - hat sich somit in den vergangenen Jahren regional erheblich ausdifferenziert und inhaltlich deutlich konkretisiert. Dennoch bleiben die von Barbara Lippert konstatierten konzeptionellen und strukturellen Defizite der ENP grundsätzlichen bestehen: „,das Oszillieren zwischen Außen- und Sicherheitspolitik, Entwicklungs- und Erweiterungspolitik, die geografische Beliebigkeit, die opake Anreizstruktur und die strategische Ambivalenz" (2008: 8). 


\section{Beyond Europe: Europäisierung durch normative Macht?}

Über ihr engeres geografisches Umfeld hinaus kooperiert die EU in einer nahezu unüberschaubaren Anzahl von Politikfeldern mit einzelnen Drittstaaten und ganzen Regionen. In jedem Einzelfall dieser als Kooperationseuropäisierung zu bezeichnenden Beziehungen herrschen spezifische Kontextbedingungen, die es in der wissenschaftlichen Erforschung näher zu ergründen gilt. In einem viel beachteten Aufsatz hat Ian Manners (2002) die These aufgestellt, bei der EU handle es sich um eine „,normative Macht“. Manners schließt an eine wissenschaftliche Auseinandersetzung zur Bestimmung des außenpolitischen Profils der EU an, die seit den 1970er Jahren zwischen den beiden Leitbildern einer Zivil- und Militärmacht pendelt (Bull 1982; Duchêne 1972). Da sich die Debatte primär auf die Frage konzentrierte, ob die EU über militärische Mittel verfügt und unter welchen Bedingungen sie diese zum Einsatz bringt, versteht Manners sein Konzept als Versuch, die Diskussion zu refokussieren. Dem Autor nach müsse der einzigartigen normativen Basis der EU stärkere Aufmerksamkeit zuteil werden. Manners Verständnis normativer Macht stellt sich wie folgt dar: „The concept of normative power is an attempt to suggest that not only is the EU constructed on a normative basis, but importantly this predisposes it to act in a normative way in world politics. It is built on the crucial, but usually overlooked observation, that the most important factor shaping the international role of the EU is not what it does or what is says, but what it is" (Manners 2002: 252). Im Kern zeichnet sich normative Macht für den Autor dadurch aus, innerhalb der Weltpolitik zu definieren, was als ,normal“ gelte (ebd.: 236). Thomas Diez hat dieses Konzept in einem eigenen Beitrag aufgegriffen und die Definition dahingehend konkretisiert, eine normative Macht verfüge über einen Einfluss darauf, was von anderen Akteuren als ,angemessenes Verhalten“ betrachtet werde (2005: 615). Mit dem Kriterium der Angemessenheit greift der Autor somit auf ein Erklärungsmoment zurück, dass Schimmelfennig und Sedelmeier im Rahmen der Europäisierungsforschung etabliert haben. Eine zusätzliche Anschlussmöglichkeit des Konzepts der normativen Macht ergibt sich dadurch, dass Manners interessanterweise eine Reihe von Kernnormen definiert, die er explizit aus dem Acquis communautaire beziehungsweise dem Acquis politique desideriert (2002: $242 \mathrm{ff}$.). Diez und Manners postulieren somit, dass die EU intern geteilte Normen nach außen externalisiert. Das Theorem der Innen-Außen-Analogie weist dabei eine frappierende Nähe zum Konzept externer Governance auf(Lavenex/Lehmkuh1/Wichmann 2009; Lavenex/Schimmelfennig 2009). Je weiter die Forschung sich somit vom Kern der Beitrittseuropäisierung entfernt, desto mehr verliert das akteurszentrierte Konditionalitätsmodell an Bedeutung und treten prozessorientierte Erklärungsan- 
sätze in den Vordergrund. Einen eindrücklichen Beweis hierfür liefert das unlängst erschienene Themenheft der Zeitschrift „West European Politics“, bei dem ein innovativer Brückenschlag von der Europäisierung hin zur Diffusion geschlagen wird (Börzel/Risse 2012).

\section{Schlussfolgerungen und Ausblick}

Dieser Beitrag hat erstens aufgezeigt, dass die Beitrittseuropäisierung in der Literatur mittlerweile verhältnismäßig gut aufgearbeitet worden ist. Dies gilt insbesondere für die Europäisierung der mittel- und osteuropäischen Staaten. Der überwiegende Anteil der Arbeiten hat sich dabei auf die Rolle der Konditionalität konzentriert und diese als zentrales Theorem herausgearbeitet. Auch zur Europäisierung Bulgariens und Rumäniens liegen bereits verschiedene Studien vor. Die hierzu bislang zutage geförderten empirischen Befunde haben die Erklärungskraft des Konditionalitätsmodells nicht grundsätzlich in Frage stellen können. Die Besonderheit des erstmalig von der EU geschaffenen Kooperations- und Kontrollverfahrens hat in seiner theoretischen Rückbindung jedoch zur Prägung des Begriffs der Post-Beitrittskonditionalität geführt.

Da es sich mit der Ausnahme Islands bei allen aktuellen und potenziellen EUBeitrittskandidaten um Länder Südosteuropas handelt, konzentrieren sich die Forschungsanstrengungen der Beitrittseuropäisierung derzeit auf die Region des westlichen Balkans und die Türkei. Obwohl die EU all diesen Staaten eine Beitrittsperspektive eingeräumt hat, erweist sich der Europäisierungsprozess hier deutlich schwieriger als erwartet. Die länderspezifischen Herausforderungen - etwa begrenzte Staatlichkeit, wirtschaftliche Rückständigkeit und fragile Stabilität - haben dabei den in der Europäisierungsforschung angelegten "domestic turn“ (Sousa 2008: 430) zusätzlich verstärkt. Detailliert werden die nationalen Bedingungen und Voraussetzungen von Europäisierungsprozessen in Südosteuropa analysiert und mit denen Mittel- und Osteuropas verglichen. Eine Übertragung des Acquis communautaire auf diese Staaten erscheint aus dieser vergleichenden Perspektive als eine zumindest weitaus langfristigere Herausforderung für die EU, ein Sachverhalt, der durch die zu konstatierende zunehmende Politisierung und Nationalisierung der EUErweiterungspolitik zusätzlich verstärkt wird.

Zweitens hat der Überblick über die aktuelle Literatur verdeutlicht, dass die Forschung keine gerade Linie von der Beitritts- zur Nachbarschaftseuropäisierung vollziehen kann. Die bislang vorliegenden Studien weisen auf eine eingeschränkte Fähigkeit der EU hin, die an der Europäischen Nachbarschaftspolitik beteiligten Staaten zu einer Übernahme europäischer Normen zu bewegen. Die durch das Feh- 
len einer klaren Beitrittsperspektive deutlich geringer ausfallende Anreizstruktur wird durch die in dieser Ländergruppe vorzufindenden multiplen Problemlagen zusätzlich belastet. Auf diese komplexe Situation hat die EU mit einer Ausdifferenzierung ihrer Politik gegenüber den Staaten der Nachbarschaft reagiert. Die Wirksamkeit dieser differenzierten Konditionalität hängt dabei jedoch von der individuellen Passfähigkeit zwischen den jeweils dargebotenen Anreizen und den länderspezifischen internen Interessenskonstellationen ab. In der Summe erweist sich die ENP somit eher als ein Katalysator für formale Europäisierung. Um diese verallgemeinernde Einschätzung zu unterstreichen, bedarf es dringend weiterer empirischer Studien. Auch theoretisch ist die Nachbarschaftseuropäisierung bislang nur in Ansätzen aufgearbeitet worden.

Der Literaturbericht hat drittens aufgezeigt, dass die Forschung zur Kooperationseuropäisierung vor einer besonders großen Herausforderung steht. Dankenswerterweise sind hierzu in den letzten Jahren vermehrt auch theoretisch orientierte Arbeiten entstanden, welche die hohe Diversität der externen Europäisierung erkennen lassen. Hierzu hat insbesondere das ,normative power Europe“-Konzept einen wichtigen Beitrag geleistet. Freilich bedarf dieses Konzept noch einer weiteren Operationalisierung. In Ermangelung eines am Acquis orientierten Konditionalitätsmodells gewinnen grundsätzlich längerfristige Lern- und Sozialisationsprozesse an Bedeutung, in denen EU-Drittstaaten europäische Normen freiwillig übernehmen und diese als ,normal“ in ihr Handlungsrepertoire übernehmen. Derartige Prozesse sind naturgemäß nur schwer messbar.

Zusammengefasst lässt sich festhalten, dass sich zwischen Beitritt, Nachbarschaft und Kooperation eine ausdifferenzierte Forschungslandschaft der externen Europäisierung etabliert hat, die über systematische und theoretisch anschlussfähige Modelle verfügt. Vor allem die sozialwissenschaftliche Diffusionsforschung, die Governance-Debatte und die Theorie des sozialen Lernens haben in der jüngeren Vergangenheit neue Blickwinkel in die wissenschaftliche Arbeit eingebracht. Gegenwärtig wird dieses Verknüpfungspotenzial in der empirischen Europäisierungsforschung noch nicht voll ausgeschöpft. Ein weiterer wichtiger Schritt wird daher darin bestehen, die empirische Basis über detaillierte Länder- und Regionalstudien weiter zu verbreitern. Jenseits von geografischen Räumen sollte sich der Fokus der Aufmerksamkeit stärker auch auf konkrete Politik- und Problemfelder beziehen. Grundsätzlich kommt der vergleichenden Perspektive jedoch eine besondere Bedeutung zu, um spezifische und zugleich auch generalisierende Erklärungsmodelle der externen Europäisierung zu entwickeln. Unter der Wendung „same but different" kann dabei ein Forschungsprogramm in die Wege geleitet werden, dass sehr anspruchsvoll, aber zugleich auch sehr Erfolg versprechend ist. 


\section{Literatur}

Altmann, Franz-Lothar, 1998: Die Balkanpolitik der EU - Regionalansatz und Prinzip der Konditionalität, in: Südosteuropa 47 (10-11), 503-515.

Altmann, Franz-Lothar, 2005: EU-Erweiterungsmüdigkeit und Westlicher Balkan (SWP-Aktuell 60), Berlin.

Avery, Graham, 2004: The Enlargement Negotiations, in: Fraser Cameron (Hrsg.), The Future of Europe. Integration and Enlargement, London, 35-62.

Axt, Heinz-Jürgen/Milošoski, Antonio/Schwarz, Oliver, 2007: Europäisierung - ein weites Feld. Literaturbericht und Forschungsfragen, in: Politische Vierteljahresschrift 28 (1), 136-149.

Biermann, Rafael, 2002: Stabilitätspakt und EU-Balkanpolitik. Von der Stabilisierung zur Integration, in: Integration 25 (3), 210-225.

Börzel, Tanja A., 2011: When Europeanization Hits Limited Statehood. The Western Balkans as a Test Case for the Transformative Power of Europe (KFG Working Paper No. 30), Berlin.

Börzel, Tanja A./Risse, Thomas, 2012: When Europeanisation Meets Diffusion: Exploring New Territory, in: West European Politics 35 (1), 192-207.

Börzel, Tanja A./Soyaltin, Digdem, 2012: Europeanization in Turkey. Stretching a Concept to its Limits? (KFG Working Paper No. 36), Berlin.

Böttger, Katrin, 2010: Die Entstehung und Entwicklung der Europäischen Nachbarschaftspolitik. Akteure und Koalitionen, Baden-Baden.

Bull, Hedley, 1982: Civilian power Europe: a contradiction in terms?, in: Journal of Common Market Studies 21 (1-2), 149-164.

Diez, Thomas, 2005: Constructing the Self and Changing Others: Reconsidering 'Normative Power Europe', in: Millennium. Journal of International Studies 33 (3), 613-636.

Duchêne, François, 1972: Die Rolle Europas im Weltsystem: Von der regionalen zur planetarischen Interdependenz, in: Max Kohnstamm/Wolfgang Hager (Hrsg.), Zivilmacht Europa - Supermacht oder Partner?, Frankfurt a. M., 11-35. Falkner, Gerda/Treib, Oliver, 2007: Three Worlds of Compliance or Four? The EU15 Compared to New Member States, Wien.

Falkner, Gerda/Treib, Oliver/Hartlapp, Miriam/Leiber, Simone, 2005: Complying with Europe. EU Harmonisation and Soft Law in the Member States, Cambridge. Franke, Anja/Gawrich, Andrea/Melnykovska, Inna/Schweickert, Rainer, 2010: The European Union's Relations with Ukraine and Azerbaijan, in: Post-Soviet Affairs $26(2), 149-183$. 
Gateva, Eli, 2010: Post-Accession Conditionality - Support Instrument for Continuous Pressure? (KFG Working Paper No. 18), Berlin.

Gawrich, Andrea/Melnykovska, Inna/Schweickert, Rainer, 2009: Neighbourhood Europeanization through ENP - The Case of Ukraine (KFG Working Paper 3), Berlin.

Gawrich, Andrea/Melnykovska, Inna/Schweickert, Rainer, 2010: Neighbourhood Europeanisation through ENP - the case of Ukraine, in: Journal of Common Market Studies 48 (5), 1209-1235.

Grabbe, Heather, 2001: How does Europeanisation affect SEE Governance? Conditionality, Diffusion and Diversity, in: Journal of European Public Policy 8 (4), 1013-1031.

Hillion, Christophe, 2010: The Creeping Nationalisation of the EU Enlargement Policy (SIEPS Report No. 6), Stockholm.

Lavenex, Sandra, 2004: EU external governance in „wider Europe“, in: Journal of European Public Policy 11 (4), 680-700.

Lavenex, Sandra, 2008: A governance perspective on the European neighbourhood policy: integration beyond conditionality?, in: Journal of European Public Policy 15 (6), 938-955.

Lavenex, Sandra/Lehmkuhl, Dirk/Wichmann, Nicole, 2009: Modes of external governance: a cross-national and cross-sectoral comparison, in: Journal of European Public Policy 16 (6), 813-833.

Lavenex, Sandra/Schimmelfennig, Frank, 2009: EU Rules Beyond Borders: Theorizing External Governance in European Politics, in: Journal of European Public Policy 16 (6), 791-812.

Levitz, Philip/Pop-Eleches, Grigore, 2009: Why No Backsliding? The EU's Impact on Democracy and Governance Before and After Accession, in: Comparative Political Studies 43 (4), 457-85.

Levitz, Philip/Pop-Eleches, Grigore, 2010: Monitoring, Money and Migrants: Countering Post-Accession Backsliding in Bulgaria and Romania, in: EuropeAsia Studies 62 (3), 461-479.

Lippert, Barbara, 2004: Glanzloser Arbeitserfolg von epochaler Bedeutung: eine Bilanz der EU-Erweiterungspolitik 1989-2004, in: dies. (Hrsg.), Bilanz und Folgeprobleme der EU-Erweiterung, Baden-Baden, 13-71.

Lippert, Barbara, 2007: Die EU-Nachbarschaftspolitik in der Diskussion - Konzepte, Reformvorschläge und nationale Positionen, Berlin/Bonn.

Lippert, Barbara, 2008: Die Europäische Nachbarschaftspolitik: viele Vorbehalte - einige Fortschritte - unsichere Perspektiven, Berlin/Bonn. 
Lippert, Barbara, 2011: EU-Erweiterung. Vorschläge für die außenpolitische Flankierung einer Beitrittspause (SWP-Studie 7), Berlin.

Manners, Ian, 2002: Normative power Europe: a contradiction in terms?, in: Journal of Common Market Studies 40 (2), 235-258.

Markov, Dimitar, 2010: The Cooperation and Verification Mechanism Three Years Later: What Has Been Done and What Is Yet to Come (FES Bulgaria Report 1), Sofia.

Noutcheva, Gergana/Bechev, Dimitar, 2008: The Successful Laggards: Bulgaria and Romania's Accession to the EU, in: East European Politics and Societies 22 (1), 114-144.

Noutcheva, Gergana/Aydin-Düzgit, Senem, 2012: Lost in Europeanisation: The Western Balkans and Turkey, in: West European Politics 35 (1), 59-78.

Pridham, Geoffrey, 2007: Romania and EU Membership in Comparative Perspective: A Post-Accession Compliance Problem? The Case of Political Conditionality, in: Perspectives on European Politics and Society 8 (2), 168-188.

Schimmelfennig, Frank/Sedelmeier, Ulrich, 2002: Theorizing EU enlargement: research focus, hypotheses, and the state of the art, in: European Public Policy 9 (4), 500-528.

Schimmelfennig, Frank/Sedelmeier, Ulrich (Hrsg.), 2005 a: The Europeanization of Central and Eastern Europe, Ithaca, NY.

Schimmelfennig, Frank/Sedelmeier, Ulrich, 2005 b: Introduction: Conceptualizing the Europeanization of Central and Eastern Europe, in: dies. (Hrsg.), The Europeanization of Central and Eastern Europe, Ithaca, NY, 1-28.

Schwarz, Oliver, 2010: Erweiterung als Überinstrument der Europäischen Union? Zur Europäisierung des westlichen Balkans seit der EU-Osterweiterung, BadenBaden.

Sousa, Maja Møller, 2008: Learning in Denmark? The Case of Danish Parliamentary Control over European Union Policy, in: Scandinavian Political Studies 31 (4), 428-447.

Spendzharova, Aneta B./Vachudova, Milada Anna, 2012: Catching Up? Consolidating Liberal Democracy in Bulgaria and Romania after EU Accession, in: West European Politics 35 (1), 39-58.

Steunenberg, Bernard/Dimitrova, Antoaneta, 2007: Compliance in the EU enlargement process: The limits of conditionality, in: European Integration online Papers 11 (5), http://eiop.or.at/eiop/texte/2007-005 a.htm (Stand: 12.3.2012).

Tolksdorf, Dominik, 2011: EU-Konditionalität bei der Polizeireform in Bosnien und Herzegowina, in: Südosteuropa 59 (4), 412-447. 
Trauner, Florian, 2009: Post-accession compliance with EU law in Bulgaria and Romania: a comparative perspective, in: European Integration online Papers 13 (2), http://eiop.or.at/eiop/texte/2009-021 a.htm (Stand: 13.3.2012).

Korrespondenzanschrift:

Dr. Oliver Schwarz

Institut für Politikwissenschaft

Universität Duisburg-Essen

Lotharstraße 65, LF 321

47057 Duisburg

E-Mail: oliver.schwarz@uni-due.de

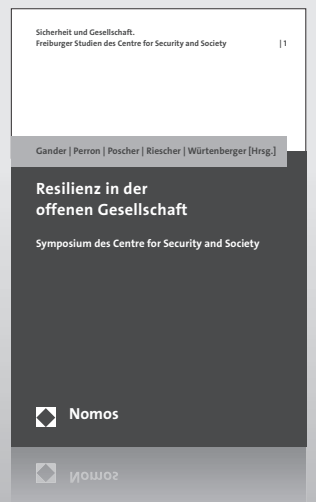

\section{Resilienz in der offenen Gesellschaft}

Symposium des Centre for Security and

Society

Herausgegeben von Hans-Helmuth Gander,

Walter Perron, Ralf Poscher, Gisela Riescher

und Thomas Würtenberger

2012, 347 S., brosch., 59,-€

ISBN 978-3-8329-7143-4

(Sicherheit und Gesellschaft. Freiburger Studien des Centre for Security and Society, Bd. 1)

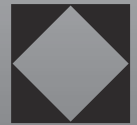

